\title{
INTRODUCTORY REMARKS
}

The subject of this Symposium is indicative of the breadth of modern astronomy in that we are to mainly consider here the nature of physical environments whose character is similar to, or more extreme than, nuclear matter. The reason for this consideration has been the discovery of those observed astronomical objects, the pulsars, and their associates, neutron stars. Here are phenomena whose explanation must ultimately be derived from the work of both astronomers and physicists of many disciplines. To quote from the concluding remarks of A. G. W. Cameron:

It is a measure of the newness of this field of dense matter calculations that probably the majority of people presenting papers are not members of the IAU. A large number of the participants are physicists who have entered the field very recently because of their interest in the properties of neutron stars. Probably Commission 35 should co-opt many of you in order to give dense matter a greater representation within the deliberations of the IAU.

On behalf of the Local Organizing Committee we should like to thank the participants in the Symposium for an enlightening and stimulating week of good science and sociality and hope that Boulder will see you again. We also thank the Graduate School, the Departments of Physics and Astrophysics and Aerospace Engineering, and The Joint Institute for Laboratory Astrophysics, all of the University of Colorado, for support both moral and financial.

Part of the time spent in editing this work was supported by NSF Grant GP-36245 through the University of Colorado. 